\title{
Reduced Emergency Department Visits and Hospitalisation with Use of an Unsanctioned Safe Consumption Site for Injection Drug Use in the United States
}

\author{
Barrot H. Lambdin, PhD ${ }^{7,2,3}$ (D), Peter J. Davidson, PhD ${ }^{4}$, Erica N. Browne, $\mathrm{MS}^{7}$, \\ Leslie W. Suen, MD' ${ }^{2}$, Lynn D. Wenger, MSW, MPH', and Alex H. Kral, PhD ${ }^{7}$ \\ 'RTI International, 2150 Shattuck Avenue, Suite 800, Berkeley, CA, USA; ${ }^{2}$ University of California San Francisco, 550 16th St, San Francisco, CA, USA; \\ ${ }^{3}$ University of Washington, 1959 NE Pacific Street, Seattle, WA, USA; “ University of California San Diego, 9500 Gillman Drive, La Jolla, CA, USA.
}

BACKGROUND: Safe consumption sites (SCS) are an evidence-based intervention to prevent drug use-related harm. In late 2014, an organisation in an undisclosed location in the USA opened an unsanctioned SCS.

OBJECTIVE: To evaluate whether use of the unsanctioned SCS affected medical outcomes.

DESIGN: A prospective cohort study.

SETTING: Neighbourhoods surrounding the SCS.

PARTICIPANTS: People who injected drugs were recruited and interviewed at baseline and 6 and 12 months from 2018 to 2020.

INTERVENTION: People using the SCS could bring preobtained drugs to consume via injection, which were monitored by trained staff with naloxone.

MAIN MEASURES: Any overdose, number of non-fatal overdoses, skin and soft tissue infections, emergency department utilisation, number of emergency department visits, hospitalisation and number of nights spent in hospital.

KEY RESULTS: A total of 494 participants enrolled in the study; 59 (12\%) used the SCS at least once. We used propensity score weighting to analyse the association between SCS utilisation and measures. People using the SCS were $27 \%$ (95\% CI: $12-46 \%$ ) less likely to visit the emergency department, had 54\% (95\% CI: 33-71\%) fewer emergency department visits, were 32\% (95\% CI: 4-57\%) less likely to be hospitalised, and spent 50\% (95\% CI: 1$85 \%)$ fewer nights in hospital. Though not significant, people using the SCS had a lower likelihood of overdosing and slightly higher likelihood of skin and soft tissue infections.

CONCLUSIONS: Our findings support the use of SCS in the USA to reduce the growing burden of acute care service utilisation related to injection drug use.

J Gen Intern Med 37(15):3853-60

DOI: $10.1007 / \mathrm{s} 11606-021-07312-4$

(c) The Author(s) under exclusive licence to Society of General Internal Medicine 2021

Received September 7, 2021

Accepted December 1, 2021

Published online January 12, 2022

\section{INTRODUCTION}

The USA has faced an epidemic of drug overdose deaths for decades.(1) The Centers for Disease Control and Prevention estimates that nearly 841,000 Americans have died of a drug overdose since 2009, and since the COVID-19 pandemic, overdose death rates have increased $30 \%$ compared to the prior year.(1,2) Since 2015, increases in opioid overdose mortality rates have been particularly pronounced among racial and ethnic minorities, rising by $114 \%$ and $97 \%$ among Black and Latinx Americans, respectively, compared with $32 \%$ among White Americans.(2)

Similarly, medical complications from injection drug use have increased in the USA.(3) Rates of new hepatitis C infections tripled from 2009 to 2018,(4) and new HIV diagnoses increased by $9 \%$ from 2014 to 2018 among people who inject drugs (PWID).(5) Cases of skin and soft tissue infections (SSTIs) from injection drug use and associated complications-abscesses, infective endocarditis and osteomyelitis - are also rising and are often life-threatening, requiring prolonged hospitalisation and complex surgeries for treatment.(3) As a result, the US health system is facing a growing burden of acute care service utilisation, including rising use of emergency response services, emergency department (ED) visits and hospitalisations to address complications stemming from injection drug use. $(6,7)$ Public health leaders are urgently exploring ways to reduce harm related to drug use.

Many instances of harm associated with drug use are preventable. For example, opioid overdose fatalities can be prevented with the timely administration of naloxone, an opioid antagonist.(8) Infectious disease transmission related to injection drug use can be prevented with use of sterile needles and syringes.(9) When SSTIs occur, early diagnosis and treatment can prevent serious complications and hospitalisations.(10) However, many PWID have limited access to these interventions, such as naloxone, or to sterile equipment that can prevent medical harm, and they often do not receive appropriate medical care until they have developed complications that require ED visits and hospitalisation.(11)

Safe consumption sites (SCS), also known as supervised injection facilities or overdose prevention sites, are an evidencebased intervention to prevent harm related to drug use, existing in 
13 countries worldwide.(12) SCS offer sterile drug use equipment and a space for people to use pre-obtained drugs under the supervision of trained staff. These staff are available to counsel PWID, administer naloxone to reverse overdoses, and refer people to primary care or substance use treatment as needed.(12) Over the past 30 years, research has shown that SCS reduce overdose mortality, prevent the spread of infectious diseases, and facilitate access to substance use treatment.(12)

However, many US legislators have been hesitant to rely on international evidence to support SCS implementation. On November 30, 2021, New York City opened the first sanctioned SCS, and Rhode Island other recently became the first US state to allow SCS https://www.nytimes.com/2021/11/30/nyregion/ supervised-injection-sites-nyc.html; https://www.wsj.com/articles/rhode-island-set-to-be-first-state-to-pilot-safe-injectionsites-for-drug-users-11635067801. While several states are pursuing legislation to authorise implementation of SCS, $(11,13)$ the pace of such efforts has been slow, in part because of a lack of US-based evidence for their effectiveness. In late 2014, an organisation in an undisclosed US urban area opened an unsanctioned SCS to address the rising medical harm from drug use in the surrounding community.(14) Using longitudinal survey data gathered from 2018 to 2020 , this study evaluated the effects of SCS use on medical outcomes, including fatal and non-fatal overdose, SSTIs, ED visits and hospitalisation.

\section{METHODS}

\section{SCS Intervention}

A description of the unsanctioned SCS has been published previously. $(14,15)$ Briefly, one unsanctioned supervised consumption site opened in September 2014, offering access by invitation only. Staff invited an initial group of people to use the SCS with the understanding that they would not disclose the site's existence to any other individuals. The number of individuals with invitations was capped at approximately 60 people due to their interest in reducing the risk of disclosure and the limited number of injection stations. If an individual stopped using the SCS, individuals who continued to use the SCS recommended new people to be invited. $(14,15)$

The SCS included six stainless steel tables (i.e. stations), operating $6-8 \mathrm{~h}$ per day for 5 days a week. At given time, approximately 50 people had invitations to the site, which were purposely limited to avoid drawing attention to the site among people in the neighbourhood and law enforcement. People who had been invited could bring pre-obtained drugs including opioids and/or stimulants to the SCS to consume via injection. All drug injection events were monitored by trained staff and conducted with provided sterile equipment at stations disinfected before each use. Syringes were used only once, after which they were discarded in on-site biohazardous waste containers. Staff were trained to attend to overdoses; naloxone, a pulse oximeter and supplemental oxygen were located on-site to help staff reverse opioid-involved overdoses. Although formal linkages with health and social service agencies were not feasible, given that the site was unsanctioned, staff provided referrals to those agencies as needed. Staff also reminded PWID about health appointments, encouraged them to seek care when needed, and in some cases accompanied them to health care visits. SCS staff were community health workers with lived experience of substance use who were trained to identify and respond to overdoses.

\section{Data Sources}

We conducted a prospective cohort study of people who inject drugs to understand the impact of the unsanctioned SCS among those who used the SCS as compared to those who did not use the SCS. In the neighbourhoods surrounding the unsanctioned SCS, we recruited PWID $(N=493)$ from 2018 to 2019 using targeted sampling methods.(16-18) Targeted sampling involved using secondary data from health and social service agencies to identify the geographic and demographic distribution of PWID in the study area. Outreach workers walked through specified recruitment areas; engaged potential study participants in conversation; and briefly described the study procedures to potentially eligible participants. Individuals interested in participating will be given a card with the hours and location of the local community field site. Upon arrival at the community field site, potential study participants were taken to a private space to meet briefly with the study coordinator, who asked a series of questions evaluating age, drug use and ability to provide informed consent to determine eligibility for the study. Eligible study participants were given an appointment, either immediately or shortly thereafter, for the informed consent process.

To be included in the study, participants had to have injected illicit drugs in the past 30 days, be 18 years of age or older, and be willing and able to provide informed consent. Drug injection was verified by visual inspection for signs of recent venepuncture (i.e. track marks). If eligible, study participants completed an informed consent process. For the informed consent, potential participants met one on one in a private room with study staff to discuss the study procedures, potential risks and benefits and the procedures the study team would employ to protect their confidentiality. Study staff also described our certificate of confidentiality, certificate \# CCDA-19-006, which protected the privacy of research subjects by prohibiting disclosure of identifiable, sensitive research information outside of the study team. If participants consented to be in the study, they were asked to provide contact information to receive upcoming visit reminders and then were administered a survey. All surveys were administered by a trained interviewer who posed questions verbally and documented participants' answers in a computer-assisted personal interviewing programme (Blaise; Statistics Netherlands, The Hague, Netherlands). Participants were surveyed at baseline and 6 and 12 months after their baseline survey. Each survey included questions regarding sociodemographic characteristics, substance use and injection practices, SCS use, overdose, HIV, hepatitis $\mathrm{C}$, health care utilisation, substance 
use treatment and law enforcement interactions. Participants were remunerated $\$ 20$ for baseline and $\$ 30$ for follow-up surveys. To promote retention, we also scheduled monthly visits to the field site between participants' survey visits, for which they were remunerated $\$ 10$.

We received data about frequency and timing of SCS utilisation for anyone who used the site within the study time period from the unsanctioned SCS. Each time an individual used drugs at the unsanctioned SCS, staff recorded the date of use and a unique identifier based on non-identifying but easily reproducible information about the individual. Those unique identifiers were also collected from participants as part of the community-based cohort study. We matched participants' information from the cohort study to SCS utilisation data to determine if and when study participants had utilised the SCS in the 6 months prior to each survey.

For cohort study participants who were lost to follow-up during the 12-month follow-up period $(N=111)$, we provided identifiers and last date seen by our study to the medical examiner's office to determine whether any of these individuals were deceased, and if so, the cause and date of death. For individuals whose identifiers matched those of a decedent, the medical examiner provided the case number, date of death, manner of death (accident, suicide, homicide, natural, undetermined) and, for all those whose manner of death was not classified as natural, a one-line cause of death. The cause was in most cases 'Acute toxicity of...' or 'Combined toxicity of...' followed by substances the medical examiner had determined to be involved in the death. If the substances listed included any type of opioid, we considered it an opioid overdose death. Types of opioids and synthetic opioids listed included fentanyl, methadone, morphine-type alkaloid and/or heroin. Natural deaths are not usually investigated by the medical examiner. For our study participants, the cause of death was not available for one decedent who was determined to have died of natural causes.

All study procedures were approved by the Institutional Review Board at the University of California San Diego. As part of this, we have agreed to take strong measures to protect the confidentiality of those involved in the study. This includes not reporting certain information that could unintentionally disclose the location of the SCS.

\section{Measures}

Outcomes. Our outcome variables were non-fatal or fatal overdose (yes/no), number of non-fatal overdoses, SSTI (yes/no), ED utilisation (yes/no), number of ED visits, hospitalisation (yes/no) and number of nights spent in hospital. To assess these outcomes, we asked about participants' experiences over the prior 6 months, such that the three semiannual surveys covered an 18-month evaluation period. Non-fatal overdose was measured as an affirmative response to 'In the last 6 months, have you had an opioid-related overdose? By overdose we mean a time when you lost consciousness, and someone had to do something to bring you back'. Fatal overdose in the past 6 months was determined with medical examiner data. For the number of non-fatal overdoses, we asked, 'In the last 6 months, how many times have you had an opioid-related overdose?' For SSTIs, participants were asked, 'In the last 6 months, have you had an abscess or other soft tissue infection related to injection drug use?' For ED utilisation, participants were asked, 'In the last 6 months, how many times have you gone to the emergency room to access health care?' For hospitalisation, participants were asked, 'In the last 6 months, how many nights have you spent in the hospital?' ED utilisation and hospitalisation were used as both dichotomous and continuous variables.

Exposure. We determined participants' SCS exposure using SCS service utilisation data. If there was at least one record of a participant having used the SCS in the 6 months (182 days) prior to a survey, the participant was considered to have been exposed to SCS during that time period. We considered the effects of any SCS use (a binary response) as opposed to a dose-response relationship, given limitations in the number of people who used the SCS and the number of times they used it. In addition, SCS use relative to drug use during the 6 months was unknown; therefore, interpretation of a continuous or categorical number of visits was unclear. Furthermore, a binary exposure mimics an intent-to-treat effect estimate typically reported in a randomised trial.

Confounders. The following measures were hypothesised a priori to be associated with SCS use. Age at enrolment was determined by date of birth and categorised as 20-29, 30-39, $40-49,50-59$ and $\geq 60$ years of age. Gender was based on self-report at enrolment and dichotomised as female (yes/no). Race and ethnicity were dichotomised based on whether participants identified as White non-Latinx (yes/no). Diagnosis of psychiatric illness (major depression, anxiety or bipolar disorder) was measured at enrolment and dichotomised (yes/no). Income from illegal activities in the past 6 months and housing stability in the past 6 months were measured at every visit and dichotomised (yes/no). We also assessed at every visit whether a participant had lived outdoors (in a tent, in a car or outside) in the past 30 days. Extensive data on participants' frequency and type of drug use, as well as alcohol use, in the past 30 days were collected during each survey. Injected opioid use, noninjected opioid use and daily alcohol use were dichotomised (yes/no). Participants were also asked during each survey whether they had used drugs in a bathroom of a social service agency in the past 6 months (yes/no); this variable was included to capture whether an individual is more trusting of or familiar with social service agencies. Finally, each survey assessed whether participants had ever participated in a substance use treatment programme (methadone, buprenorphine, 
or naltrexone; yes/no) and if they were currently enrolled in treatment (yes/no).

\section{Analytic Approach}

Because a randomised controlled trial was not a feasible approach to assess the impact of an unsanctioned SCS, we employed a quasi-experimental, longitudinal design using propensity scores. Propensity score methods such as inverse probability of treatment weighting (IPTW) have been shown to be an effective strategy for removing selection bias and simulating the results of randomised controlled trials.(19) Weights based on variables hypothesised to be associated with exposure are applied so that participants who did not use the SCS have approximately similar characteristics to those who did (i.e. balancing covariates as we would expect under randomisation).

IPTW was also used to account for time-varying confounding.(20) Weights were created from covariate balancing propensity scores (CBPS),(21) which optimise covariate balance as well as prediction of exposure. The probability of SCS use in the prior 6 months (yes/no) was estimated using a pooled logistic regression model. This approach created weights for each participant at each interview, ensuring balance across all hypothesised a priori confounding variables at every time point. Time-fixed confounders (assessed at baseline) included age category, female gender, White non-Latinx race/ethnicity and psychiatric illness diagnosis. Time-varying confounders were determined from each visit and included being unstably housed; currently living outdoors; any use of injected opioids, any use of non-injected opioids or alcohol use every day in the past month; currently enrolled or ever enrolled in a medication-assisted treatment programme; income from illegal activities; and ever having used drugs in a social service agency bathroom. Those who had used the SCS in the prior 6 months were assigned a weight of 1 , and those who had not were given a weight based on their CBPS. Thus, effect estimates represent the average treatment effect on the treated.

Because the SCS is unsanctioned, we cannot report the prevalence of variables used in our study, which might indirectly disclose the location of the SCS. Instead, we provide information regarding the differences in potential confounding variables between those who did and did not use the SCS, before and after weighting.
To compare outcomes between those who did and did not use the SCS, we used Poisson models with a log link function to estimate risk ratios for binary outcomes and incident rate ratios for count outcomes. Regarding SCS exposure, we hypothesised that SCS use would be concurrently associated with our outcomes within the same 6-month timeframe. Generalised estimating equations with an unstructured correlation matrix were used to account for repeated measures over the study period. Bootstrap confidence intervals were calculated using percentiles from 1000 replicate samples (with replacement) from the observed data.(22) Our interpretation of effect estimates focuses on the point estimate and $95 \%$ confidence interval rather than statistical significance at a designated $p$ value threshold. CBPS were estimated using the WeightIt package in $\mathrm{R} ;(23)$ all analyses were conducted using $\mathrm{R}$ version 4.0.2.

\section{Role of the Funding Source}

Arnold Ventures supported this work. Arnold Ventures had no role in the design or conduct of the study; collection, management, analysis or interpretation of the data; preparation, review or approval of the manuscript; or decision to submit the manuscript for publication.

\section{RESULTS}

A total of 494 participants enrolled in the study; of those, 405 (82\%) completed their 6-month visit and 385 (78\%) completed their 12-month visit. Of the 111 participants lost to followup, 13 died, including 11 who died from an opioid-involved overdose during the study. No one died at the SCS.

Fifty-nine (12\%) of the 494 participants used the SCS at least once during the three 6-month observation periods. Across each 6-month period, those who used the SCS visited a median of 18 times (IQR 6-43 visits, range 1-199 visits) (Table 1). Most people who used the SCS did so inconsistently. Although we were unable to assess how many of a participant's drug injection events occurred at the SCS during each 6-month period, we could estimate the proportion based on reported injections in the prior month. Among those who used the SCS in the month before each interview, on average

Table 1 Summary of Safe Consumption Site Use in the 6 Months Prior to Each Survey

\begin{tabular}{|c|c|c|c|}
\hline \multirow[t]{2}{*}{ Summary statistic } & \multicolumn{3}{|l|}{ Study visit } \\
\hline & $\begin{array}{l}\text { Baseline } \\
(N=493)\end{array}$ & $\begin{array}{l}\text { 6-month } \\
(N=404)\end{array}$ & $\begin{array}{l}\text { 12-month } \\
(N=382)\end{array}$ \\
\hline Visited site at least once $-N(\%)$ & $50(10 \%)$ & $49(12 \%)$ & $45(12 \%)$ \\
\hline \multicolumn{4}{|l|}{ Among those who used the site, number of visits } \\
\hline Mean (standard deviation) & $25.9(27.4)$ & $34.4(38.1)$ & $29.9(28.5)$ \\
\hline Median (interquartile range) & $15.5(6-43)$ & $22(4-49)$ & $26(6-38)$ \\
\hline Range & $1-105$ & $1-199$ & $1-130$ \\
\hline
\end{tabular}


Table 2 Percentage Differences in Potential Confounders at Baseline and Before and After Weighting

\begin{tabular}{|c|c|c|}
\hline Baseline characteristics & Before weighting (\% difference) & After weighting (\% difference) \\
\hline \multicolumn{3}{|l|}{ Age at enrolment (years), category } \\
\hline $20-29$ & 1 & 5 \\
\hline $30-39$ & 18 & -1 \\
\hline $40-49$ & -5 & -3 \\
\hline $50-59$ & -9 & 2 \\
\hline$\geq 60$ & -6 & -2 \\
\hline Female & -10 & 3 \\
\hline White, non-Latinx & 21 & 0 \\
\hline Unstable housing & 5 & 0 \\
\hline Living outdoors & 14 & 4 \\
\hline Injected opioids, past 30 days & 8 & 0 \\
\hline Non-injection use of opioids, past 30 days & -3 & 0 \\
\hline Daily alcohol use, past 30 days & -6 & -3 \\
\hline Ever enrolled in a treatment programme & 14 & 5 \\
\hline Currently enrolled in a treatment programme & -10 & 0 \\
\hline Income from illegal activities & 22 & -2 \\
\hline Psychiatric illness diagnosis & -5 & -1 \\
\hline Ever used drugs in bathroom of service agency & 15 & -3 \\
\hline
\end{tabular}

$\%$ difference $=$ those who used the safe consumption site in 6 months prior to enrolment - those who did not use the site in 6 months prior to enrolment. Positive values indicate greater frequency of a covariate (or category) among people using the safe consumption site compared to those not using the safe consumption site at enrolment

$8 \%$ of drug injection events were at the SCS (median $4 \%$, IQR 2-10\%).

Before we applied CBPS weights, participants who used the SCS differed on hypothesised confounding variables including age, gender, race, income and medication-assisted treatment experience. After weighting, people who used the SCS and who did not use the SCS were well balanced, with $\leq 5 \%$ difference between the two groups for all included measures (Table 2).

People using the SCS had a $24 \%$ lower risk of any overdose (fatal or non-fatal) within the same 6-month period, although the estimated relative risk was not statistically significant (relative risk [RR] 0.76, 95\% CI: 0.52-1.22; Table 3). Though the differences were not statistically significant, people using the SCS had a $13 \%$ lower incident rate of non-fatal overdose (incident rate ratio [IRR]: $0.87,95 \%$ CI $0.49-1.69$ ) and a $14 \%$ higher risk of SSTIs compared with those who were not using the SCS (RR 1.14, 95\% CI: 0.82-1.41).

Health care utilisation differed by SCS use (Table 4). Compared with participants who were not using the SCS, those who were using the SCS were $27 \%$ less likely to visit the ED (95\% CI 12-46\%), had 54\% fewer ED visits (95\% CI 33$71 \%$ ), were $32 \%$ less likely to be hospitalised (95\% CI 4 $57 \%$ ), and spent $50 \%$ fewer nights hospitalised (95\% CI 1$85 \%)$.

\section{DISCUSSION}

We report on the first community-based, quasi-experimental cohort study evaluating the impact of an unsanctioned SCS in the USA. We found that the likelihood of visiting the ED, frequency of ED visits, likelihood of hospitalisation and number of hospitalised nights were significantly lower among people who were using the SCS than people who were not. These findings support the use of SCS to reduce the growing burden of acute care service utilisation related to injection drug use in the USA.

There are two possible explanations for these findings. First, participants who experienced opioid-involved overdoses at the SCS could be treated with naloxone and monitored onsite, preventing the need for overdose-related ED visits.(14) Second, although no licensed, board-certified health professional was working on-site, staff at the SCS could have triaged participants and referred those with SSTIs to primary care before complications developed, thereby reducing the need for ED visits and hospitalisations. In any case, participants' use of the SCS may have reduced preventable medical expenses, including overdose-related ED visits, overdose-related hospitalisations and SSTI hospitalisations.(12, 24, 25) If the SCS were sanctioned, we would expect more direct relationships with primary care services, perhaps offering some on-

Table 3 Estimated Associations of Safe Consumption Site Use with Overdoses and Skin and Soft Tissue Infections

\begin{tabular}{llll}
\hline \hline Outcome & Effect estimate & $\begin{array}{c}\text { Bootstrap } \\
\mathbf{( 9 5 \%} \mathbf{C I )}\end{array}$ \\
\hline Overdose, non-fatal or fatal (any) - $\mathbf{R R}$ & 0.76 & 0.87 \\
Overdose, non-fatal (count)-IRR & 1.14 & $(0.52-1.22)$ \\
Skin and soft tissue infection (any) - $\mathbf{R R}$ & $(0.49-1.69)$ \\
\hline
\end{tabular}

Analysis covers the 18-month evaluation period and 1267 individual SCS visits

$I R R$ incident rate ratio, $R R$ relative risk 
Table 4. Estimated Associations of Safe Consumption Site Use with Emergency Department Visits and Hospitalisation

\begin{tabular}{llr}
\hline \hline Outcome & Effect estimate & $\begin{array}{c}\text { Bootstrap } \\
\mathbf{( 9 5 \%} \mathbf{C I})\end{array}$ \\
\hline Emergency department visit (any)_RR & & $(0.54-0.88)$ \\
Emergency department visits (count)_-IRR & 0.73 & $(0.29-0.67)$ \\
Hospitalisation (any)_-RR & 0.46 & $(0.43-0.96)$ \\
Hospitalised nights (count)_IRR & 0.68 & $(0.15-0.99)$ \\
\hline
\end{tabular}

Analysis covers the 18-month evaluation period and 1267 individual SCS visits

$I R R$ incident rate ratio, $R R$ relative risk

site medical care, as is done in many SCS globally. These findings align with results from a longitudinal, ecological study from Australia that showed a reduction in emergency service use at hospitals located near an SCS.(26) However, our study is the first to document reductions in ED visits and hospitalisations among people using an SCS.

Our results suggest that people using the SCS may be somewhat less likely to experience a fatal or non-fatal overdose and have fewer non-fatal overdoses, but results were not statistically significant. Findings from research in Vancouver, Canada, showed a 35\% reduction in the fatal overdose death rate in the area immediately surrounding a sanctioned SIF as compared to other areas of the city.(27) When considering these findings, it is important to keep in mind several structural factors. First, most injection events occurred outside of the SCS; even among participants who used the SCS, the proportion of on-site drug injection events was relatively low. Of the 11 participants who died from an overdose during the study, all occurred among people who had never used or were currently not using the SCS. Furthermore, all overdoses that occurred at the SCS were successfully reversed.(14) If the SCS were sanctioned, more people could have used it and a greater proportion of their injections could have taken place there, further reducing the likelihood of overdose deaths. However, increases in overdoses and overdose deaths in the USA over the past 5-10 years have been due in part to fentanyl contamination in the illicit drug supply.(28, 29) Although the SCS provided a place for supervised, unrushed consumption and staff who could treat an overdose if it occurred, it could not prevent overdoses due to contaminated drugs. SCS can potentially reduce those overdose risks by offering point-of-care drug-testing capabilities that identify and quantify substances within a participant's drug.(29)

We also found that people using the SCS had a slightly elevated risk of SSTIs, but results were not statistically significant. These findings are similar to results from two studies in Germany and Canada that did not show statistically significant SSTI changes with SCS use in a sanctioned environment. $(30,31)$ However, a recent review noted that an SCS could facilitate earlier diagnosis and treatment of SSTIs because, as staff build relationships with participants, they can carry out more screenings for SSTIs, refer participants to community health clinics for treatment, and support participants through follow-up.(12) It follows that this could yield earlier and more efficient movement through the continuum of care for SSTIs, thereby preventing medical complications and the subsequent need for ED visits and hospitalisations.

Our results should be considered in light of potential limitations. First, our study was a prospective cohort study in which exposure to SCS was not randomised. Our analytic approach - weighting based on CBPS - is a robust, gold standard approach to minimise bias due to self-selection when the intervention under study cannot be randomised. However, a limitation with this approach is that other unmeasured confounders that we did not account for in our propensity score model could bias our results. Second, only $12 \%$ of study participants used the SCS, and most of those did so for a small proportion of their injections. This prevented us from having sufficient statistical power to assess a dose-response effect. However, we anticipate that our approach (i.e. considering someone as exposed to the SCS if they visited at least once in the past 6 months) would bias our results toward the null. Future studies of a sanctioned SCS with higher levels of utilisation would be able to estimate effects based on amount of utilisation. Third, we could not determine the ordering of events within each 6-month period, limiting our ability to make causal associations. Fourth, most of our study variables were self-reported and could reflect response biases attributable to recall error or social desirability. Yet we expect any resulting misclassification to be nondifferential with respect to SCS use, which would also bias our results toward the null, on average. Moreover, we did not have the resources to verify reported ED visits and hospitalisations with administrative data from hospitals, and we did not have information on whether the participant went to a public or private hospital. However, we were able to collect SCS use using administrative data from the SCS and fatal overdose events from the medical examiner.

In conclusion, we present the first community-based cohort study evaluating the impact of an unsanctioned SCS on 
medical outcomes in the USA. People using the SCS were less likely to visit the ED, visited the ED less often, were less likely to be hospitalised, and spent fewer nights in hospital. These findings are encouraging, given the low levels of SCS use attributable to the SCS's unsanctioned nature. Our results suggest that SCS might be an important component of a comprehensive approach to address the rising levels of medical harm related to injection drug use and the resultant burden on the medical system.

Acknowledgements: This manuscript was drafted by BHL, ENB and LWS; further edited by AHK, PJD and LDW; and approved by all authors. ENB, BHL and AHK vouch for the data and analyses, and for the fidelity of this report to the data analysis plan. BHL, AHK, PJD and LDW contributed to conceptualisation, methodology development, funding acquisition and data collection. ENB contributed to data management and performed the data analysis in collaboration with BHL, AHK, PJD and LDW. All authors contributed to data interpretation and critical review of the manuscript. The authors have no conflicts of interest to declare. We would like to thank Arnold Ventures for supporting this work. Specifically, we would like to thank Julie Wiegandt and Kirby Smith for their contributions. We would like to acknowledge all of our study participants, as well as the people who staffed the unsanctioned SCS.

Corresponding Author: Barrot H. Lambdin, PhD; RTI International, 2150 Shattuck Avenue, Suite 800, Berkeley, CA 94704, USA (e-mail: blambdin@rti.org).

Funding Arnold Ventures

\section{Declarations:}

Conflict of Interest: The authors declare that they do not have a conflict of interest.

Data sharing: To protect the confidentiality of the SCS and its participants, we cannot make these data public at this time.

\section{REFERENCES}

1. Ahmad FB, Rossen LM, Sutton P. Provisional drug overdose death counts. National Center for Health Statistics. 2021.

2. Centers for Disease Control and Prevention, National Center for Health Statistics. Wide-ranging Online Data for Epidemiologic Research (WONDER) 1999-2019. Centers for Disease Control and Prevention. Available at: https://wonder.cdc.gov/ucd-icd10.html. Accessed May 26, 2021.

3. Levitt A, Mermin J, Jones CJ, See I, Butler JC. Infectious diseases and injection drug use: public health burden and response. $J$ Infect Dis. 1 October 2020 2020;222(Supplement 5):S213-S217. doi: https://doi.org/ 10.1093/infdis/jiaa432

4. Ryerson AB, Schillie S, Barker LK, Kupronis BA, Wester C. Vital signs: newly reported acute and chronic Hepatitis C Cases - United States, 2009-2018. MMWR Morb Mortal Wkly Rep. Apr 10 2020;69(14):399-404. doi:https://doi.org/10.15585/mmwr.mm6914a2

5. Centers for Disease Control and Prevention. HIV in the United States: At a glance. Available at: https://www.cdc.gov/hiv/statistics/overview/ ataglance.html. Accessed April 16, 2021.

6. Singh JA, Cleveland JD. National U.S. time-trends in opioid use disorder hospitalizations and associated healthcare utilization and mortality. PLoS One. 2020;15(2):e229174. doi:https://doi.org/10.1371/journal.pone. 0229174

7. Holland KM, Jones C, Vivolo-Kantor AM, et al. Trends in US emergency department visits for mental health, overdose, and violence outcomes before and during the COVID-19 pandemic. JAMA Psychiatry. Apr 1 2021;78(4):372-379. doi:https://doi.org/10.1001/jamapsychiatry.2020. 4402

8. Walley AY, Xuan Z, Hackman HH, et al. Opioid overdose rates and implementation of overdose education and nasal naloxone distribution in
Massachusetts: interrupted time series analysis. BMJ. 2013;346:f174. doi:https://doi.org/10.1136/bmj.f174

9. Watters JK, Estilo MJ, Clark GL, Lorvick J. Syringe and needle exchange as HIV/AIDS prevention for injection drug users. JAMA. Jan 12 1994;271(2):115-20

10. Centers for Disease Control and Prevention. Soft tissue infections among injection drug users-San Francisco, California, 1996-2000. MMWR Morb Mortal Wkly Rep 18 May 2001 2001;50(19):381-4.

11. Beletsky L, Davis CS, Anderson E, Burris S. The law (and politics) of safe injection facilities in the United States. Am $J$ Public Health. Feb 2008;98(2):231-7. doi:https://doi.org/10.2105/AJPH.2006.103747

12. Armbrecht E, Guzauskas G, Hansen R, et al. Supervised injection facilities and other supervised consumption sites: effectiveness and value; final evidence report. 2021May 27. https://icer.org/wp-content/uploads/ 2020/10/ICER SIF Final-Evidence-Report 010821.pdf

13. Kennedy-Hendricks A, Bluestein J, Kral AH, Barry CL, Sherman SG. Establishing sanctioned safe consumption sites in the United States: five jurisdictions moving the policy agenda forward. Psychiatr Serv. Apr 1 2019;70(4):294-301. doi:https://doi.org/10.1176/appi.ps.201800398

14. Kral AH, Lambdin BH, Wenger LD, Davidson PJ. Evaluation of an unsanctioned safe consumption site in the United States. $N$ Engl J Med. Aug 6 2020;383(6):589-590. doi:https://doi.org/10.1056/ NEJMc2015435

15. Davidson PJ, Lambdin BH, Browne EN, Wenger LD, Kral AH. Impact of an unsanctioned safe consumption site on criminal activity, 2010-2019. Drug Alcohol Depend. Mar 1 2021;220:108521. doi:https://doi.org/10. 1016/j.drugalcdep.2021.108521

16. Watters JK, Biernacki P. Targeted sampling: options for the study of hidden populations. Soc Probl. 1989;36(4):416-430. doi:https://doi.org/ $10.2307 / 800824$

17. Bluthenthal RN, Watters JK. Multimethod research from targeted sampling to HIV risk environments. NIDA Res Monogr. 1995;157:212-30.

18. Kral AH, Malekinejad M, Vaudrey J, et al. Comparing respondent-driven sampling and targeted sampling methods of recruiting injection drug users in San Francisco. J Urban Health. Sep 2010;87(5):839-50. doi:https://doi.org/10.1007/s11524-010-9486-9

19. Austin PC. An introduction to propensity score methods for reducing the effects of confounding in observational studies. Multivariate Behav Res. May 2011;46(3):399-424. doi:https://doi.org/10.1080/00273171.2011. 568786

20. Austin PC, Stuart EA. Moving towards best practice when using inverse probability of treatment weighting (IPTW) using the propensity score to estimate causal treatment effects in observational studies. Stat Med. 2015;34(28):3661-79. doi:https://doi.org/10.1002/sim.6607

21. Imai K, Ratkovic M. Covariate balancing propensity score. Journal of the Royal Statistical Society, Series B (Statistical Methodology). 2014;76(1):243-46.

22. Effron B, Tibshirani R. Bootstrap methods for standard errors, confidence intervals and other measures of statistical accuracy. Stat Sci. 1986;1:5477.

23. Greifer N. WeightIt: weighting for covariate balance in observational studies. 2020. https://cran.r-project.org/web/packages/WeightIt/ WeightIt.pdf

24. Capizzi $\mathrm{J}$, Leahy $\mathrm{J}$, Wheelock $\mathrm{H}$, et al. Population-based trends in hospitalizations due to injection drug use-related serious bacterial infections, Oregon, 2008 to 2018. PLoS One. 2020;15(11):e0242165. doi:https://doi.org/10.1371/journal.pone.0242165

25. Armbrecht E, Guzauskas G, Hansen R, et al. Supervised Injection Facilities and Other Supervised Consumption Sites: Effectiveness and Value; Final Evidence Report. Institute for Clincal and Economic Review. https://icerorg/wp-content/uploads/2020/10/ICER_SIF_Final-EvidenceReport_010821pdf (Accessed May 27, 2021). 2021;

26. KPMG. Further evaluation of the medically supervised injecting centre during its extended trial period (2007-2011). Available at: http://www. directionsact.com/pdf/drug_news/MISC_evaluation.pdf. Accessed May 3, 2021.

27. Marshall BD, Milloy MJ, Wood E, Montaner SJ, Kerr T. Reduction in overdose mortality after the opening of North America's first medically supervised safer injecting facility: a retrospective population-based study. Lancet. Apr 2011;377:9775:1429-37. doi: https://doi.org/10.1016/ S0140-6736(10)62353-7

28. Ciccarone D. Fentanyl in the US heroin supply: a rapidly changing risk environment. Int $J$ Drug Policy. Aug 2017;46:107-111. doi:https://doi. org/10.1016/j.drugpo.2017.06.010

29. Ciccarone D, Unick GJ, Cohen JK, Mars SG, Rosenblum D. Nationwide increase in hospitalizations for heroin-related soft tissue infections: 
associations with structural market conditions. Drug Alcohol Depend Jun 1 2016;163:126-33. doi:https://doi.org/10.1016/j.drugalcdep. 2016.04.009

30. Lloyd-Smith E, Wood E, Zhang R, Tyndall MW, Montaner JS, Kerr T. Risk factors for developing a cutaneous injection-related infection among injection drug users: a cohort study. BMC Public Health. Dec 9 2008;8:405. doi:https://doi.org/10.1186/1471-2458-8-405

31. Scherbaum N, Specka M, Schifano F, Bombeck J, Marrziniak B. Longitudinal observation of a sample of German drug consumption facility clients. Subst Use Misuse. 2010;45(1-2):176-89. doi:https://doi. org/10.3109/10826080902873044

Publisher's Note: Springer Nature remains neutral with regard to jurisdictional claims in published maps and institutional affiliations. 